Internist 2021 - 62:679-685

https://doi.org/10.1007/s00108-021-01021-0

Angenommen: 9. März 2021

Online publiziert: 16. April 2021

(c) Der/die Autor(en) 2021

\section{Redaktion}

M. Wehling, Mannheim

Horst Olschewski' • Roland Buhl ${ }^{2} \cdot$ Georg Christian Funk ${ }^{3} \cdot$ Arschang Valipour ${ }^{4} \cdot$ Claus F. Vogelmeier

'Klinische Abteilung für Pulmonologie, Universitätsklinik für Innere Medizin, Medizinische Universität Graz, Graz, Österreich

${ }^{2}$ Schwerpunkt Pneumologie, III. Medizinische Klinik und Poliklinik, Universitätsmedizin der Johannes Gutenberg-Universität Mainz, Mainz, Deutschland

${ }^{3}$ 2. Medizinische Abteilung mit Pneumologie mit AmbulanzKarl Landsteiner Institut für Lungenforschung und Pneumologische Onkologie, Klinik Ottakring, Wien, Österreich

${ }^{4}$ Abteilung für Innere Medizin und Pneumologie, Karl-Landsteiner-Institut für Lungenforschung und Pneumologische Onkologie, Klinik Floridsdorf, Wien, Österreich

${ }^{5}$ Klinik für Innere Medizin, Pneumologie, Intensiv- und Schlafmedizin, Universitätsklinikum Gießen und Marburg, Standort Marburg, Marburg, Deutschland

\title{
Chronisch-obstruktive Lungenerkrankung 2021 - die richtige Therapie für den richtigen Patienten
}

Bronchodilatation weiterhin die Grundlage der COPD-Therapie. Für Patienten mit gehäuften Exazerbationen („frequent exacerbators") bedeutet eine Triple-Therapie mit LAMA+LABA+ICS einen Mortalitätsvorteil. Weitere Analysen oder Studien sollen klären, ob das für spezifische Subgruppen stärker ausgeprägt ist.

\section{Differenzialdiagnosen zur Exazerbation}

Eine Exazerbation ist definiert als eine akute Verschlechterung der respiratorischen Symptome, die zu einer Steigerung der Therapie führt [9]. Doch nicht jede Verschlechterung kommt durch eine Exazerbation zustande. Denken Sie nur an Ihren 70-jährigen Patienten mit Parkinson-Erkrankung, der rezidivierend aspiriert und dadurch wiederholt Pneumonien entwickelt, oder an Ihre 80-jährige Patientin, die immer wieder hypertensive Krisen hat und dann mehr Medikamente braucht. Der GOLD-Report listet eine Vielzahl von Krankheiten als mögliche Differenzialdiagnosen einer Exazerbation auf (• Tab. 1). Vielfach unterschätzt wird die akute Lungenembolie, die einer aktuellen Studie zufolge in $6 \%$ der Fäl-

\begin{tabular}{|c|c|}
\hline $\begin{array}{l}\text { Differenzial- } \\
\text { diagnose }\end{array}$ & Diagnostik \\
\hline \multirow[t]{2}{*}{ Pneumonie } & $\begin{array}{l}\text { Röntgenuntersuchung } \\
\text { des Thorax }\end{array}$ \\
\hline & $\begin{array}{l}\text { C-reaktives Protein und/ } \\
\text { oder Prokalzitonin }\end{array}$ \\
\hline Pneumothorax & $\begin{array}{l}\text { Röntgenuntersuchung } \\
\text { des Thorax oder Sono- } \\
\text { graphie }\end{array}$ \\
\hline Pleuraerguss & $\begin{array}{l}\text { Röntgenuntersuchung } \\
\text { des Thorax oder Sono- } \\
\text { graphie }\end{array}$ \\
\hline \multirow[t]{2}{*}{ Lungenembolie } & $\begin{array}{l}\text { D-Dimer und/oder } \\
\text { Venenduplex }\end{array}$ \\
\hline & $\begin{array}{l}\text { Computertomo- } \\
\text { graphische Pulmona- } \\
\text { lisangiographie }\end{array}$ \\
\hline \multirow[t]{2}{*}{$\begin{array}{l}\text { Kardiogenes Lun- } \\
\text { genödem }\end{array}$} & $\begin{array}{l}\text { Elektrokardiogramm } \\
\text { und Echokardiogramm }\end{array}$ \\
\hline & Herzenzyme \\
\hline $\begin{array}{l}\text { Kardiale Arrhyth- } \\
\text { mie - Vorhofflim- } \\
\text { mern/-flattern }\end{array}$ & Elektrokardiogramm \\
\hline
\end{tabular}




\section{Arzneimitteltherapie}

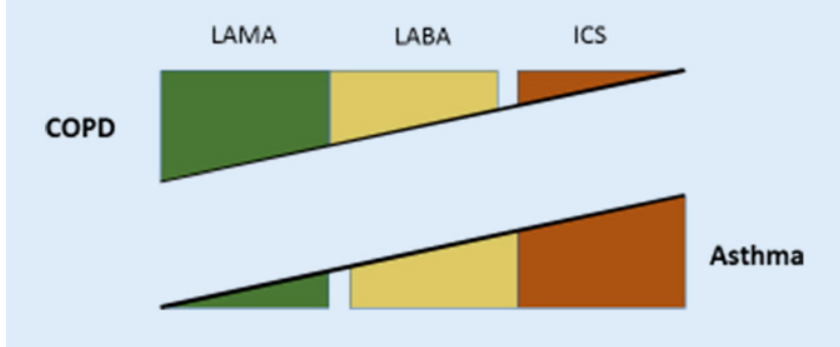

Abb. 1 A Evidenz für Substanzgruppen bei chronisch-obstruktiver Lungenerkrankung und Asthma. Substanzgruppen: ICS inhalative Kortikosteroide, LABA "long-acting $\beta$ agonists" (lang wirksame $\beta$-Mimetika), LAMA „long-acting muscarinic antagonists“ (lang wirksame Muskarinantagonisten). Die GröBe der Felder spiegelt die Evidenz für die entsprechende Substanzgruppe wider. Die Nähe der Felder zueinander symbolisiert die Evidenz für entsprechende Kombinationstherapien

le der respiratorischen Verschlechterung zugrunde liegt [10].

\section{Bronchodilatation als Basistherapie}

Es gibt sehr gute Evidenz, dass eine duale Bronchodilatation mit einem LAMA und LABA im Vergleich zu einer Monotherapie zu einer verbesserten Lungenfunktion und Lebensqualität führt, ohne dass dabei vermehrte Nebenwirkungen auftreten [11]. Es herrschte bisher aber die Meinung vor, das wäre für COPD-Patienten mit hoher Beschwerdelast wichtiger als für Patienten mit geringer Beschwerdelast. Die EMAX-Studie [12] hat bei COPD-Patienten mit einer mittleren Einsekundenkapazität („forced expiratory volume in $\left.1 \mathrm{~s}^{\text {" }}\left[\mathrm{FEV}_{1}\right]\right)$ von $55 \%$ der Norm und COPD-AssessmentTest(CAT)-Scores zwischen 10 und 40 (im Mittel 20) untersucht, wie die Vorteile der dualen Bronchodilatation vs. Monotherapie vom CAT-Score abhängen. Das Besondere der EMAX-Studie war, dass in keinem der Studienarme ICS zum Einsatz kamen. Diese Studie zeigte, dass der Nutzen der dualen Bronchodilatation weitgehend unabhängig vom CAT-Score war und nominell der größte Vorteil im CATBereich zwischen 10 und 20 zu finden war [13]. Das kann als Argument dafür dienen, alle symptomatischen Patienten mit COPD (CAT-Score $\geq 10$ ) von Anfang an mit einer dualen Bronchodilatation zu behandeln.

\section{Unterschiede zwischen Asthma und chronisch-obstruktiver Lungenerkrankung}

Die Therapie der COPD unterscheidet sich grundsätzlich von jener des Asthma bronchiale. Im Zentrum der COPDTherapie steht die Bronchodilatation mit einem LAMA, während für das Asthma das ICS die Basis der Therapie darstellt. LABA spielen als Kombinationspartner sowohl bei der COPD als auch beim Asthma eine wichtige Rolle (• Abb. 1).

\section{Triple-Therapie}

Sowohl bei der COPD als auch beim Asthma bronchiale wurden in der letzten Zeit Triple-Therapien (LAMA + LABA + ICS) getestet, allerdings unter unterschiedlichen Vorzeichen. Beim Asthma bronchiale verbesserte das zusätzliche LAMA im Wesentlichen die Lungenfunktion [14], bei der COPD verbesserte das zusätzliche ICS die Zahl der Exazerbationen. Es gibt bei COPD auch Hinweise auf einen reduzierten Abfall der $\mathrm{FEV}_{1}$ pro Jahr unter inhalativer Therapie, was auf die Effekte von LAMA und ICS zurückzuführen ist [15].

\section{Praktische Konsequenzen}

Unter Berücksichtigung der Ergebnisse der großen Triple-Therapie-Studien [3, 4, 6], der aktuellen Daten der EMAXStudie $[12,13]$ und der Interaktion mit dem Zigarettenrauchen [16] ergeben sich in Übereinstimmung mit dem GOLDReport 2021 [9] für COPD (a) und dem Global-Initiative-for-Asthma(GINA)-
Update 2020 [17] für Asthma (b) folgende Differenzialindikationen:

- LAMA-Monotherapie:

a. Für oligosymptomatische COPDPatienten (CAT-Score < 10)

b. Keine Indikation für Asthma bronchiale

- LAMA+ LABA:

a. Für alle symptomatischen COPDPatienten (CAT-Score $\geq 10$ )

b. Keine Indikation für Asthma bronchiale

- ICS-Monotherapie:

a. Keine Indikation für COPD

b. Für Patienten mit Asthma bronchiale, die damit eine kontrollierte Erkrankung erreichen

- ICS + LABA:

a. Für oligosymptomatische COPDPatienten mit vermehrten Exazerbationen, insbesondere wenn folgende Randbedingungen vorliegen: > 300 Eosinophile/ $\mu$ l Blut, Asthmaanamnese oder begleitendes Asthma

b. Für Patienten mit Asthma, die unter ICS-Monotherapie keine kontrollierte Erkrankung erreichen, oder in Form von ICS + Formoterol als Bedarfstherapie in den Stufen GINA 1 und 2

- ICS + LABA + LAMA:

a. Für symptomatische COPDPatienten mit vermehrten Exazerbationen trotz einer Therapie mit ICS + LABA oder LABA + LAMA

b. Für Patienten mit Asthma, die unter ICS + LABA keine Asthmakontrolle erreichen

In - Abb. 2 sind Überlegungen zusammengefasst, die zu Beginn einer ICS-Therapie angestellt werden sollten $[9,18]$.

\section{Senkung der Mortalität von COPD-Patienten durch Triple- Therapie - Mythos oder Realität?}

Die 3-armige IMPACT-Studie $(n=$ 10.355) untersuchte COPD-Patienten mit einer hohen Exazerbationsrate und randomisierte sie auf LABA + LAMA vs. ICS + LABA vs. ICS + LABA + LAMA. Der primäre Endpunkt war die Exazerbationsrate. Die Mortalität im Triple- 
Arm (und im LABA/ICS-Arm) war signifikant niedriger als im LABA/LAMAArm [4]. Die Mortalität war allerdings nicht der primäre Endpunkt der Studie. Eine Post-hoc-Analyse, in der alle Mortalitätsereignisse erfasst und analysiert wurden, bestätigte die erniedrigte Mortalität im Triple-Therapie-Arm [7]. Allerdings war der beobachtete Effekt auf Patienten beschränkt, die bereits vor Einschluss in die Studie eine ICS-haltige Therapie erhalten hatten (s. unten).

Die 4-armige ETHOS-Studie ( $n=$ 8509) untersuchte die Exazerbationsrate bei COPD-Patienten mit einer hohen Exazerbationsrate (ICS + LABA vs. LABA + LAMAvs. niedrig dosiertes ICS + LABA + LAMA vs. hoch dosiertes ICS + LABA + LAMA). Der Therapiearm mit der höheren ICS-Dosis $(n=2137)$ in der TripleKombination zeigte im Vergleich mit der LABA + LAMA-Kombination eine geringere Mortalität [6]. Hier gab es keinen Unterschied zu LABA + ICS, es machte aber auch keinen Unterschied, ob die Patienten vor Studienbeginn eine ICS-haltige Therapie erhalten hatten oder nicht [8].

Umstritten ist bislang, welche Rolle das Absetzen von ICS in der Gruppe von Patienten gespielt haben könnte, die in den Studien mit LABA + LAMA behandelt wurden. So haben Suissa $u$. Ariel [19] eine Analyse der monatlichen Exazerbationsraten in IMPACT und TRIBUTE vorgelegt, wonach Unterschiede zwischen dem Triple-Arm und dem LABA + LAMA-Arm nur in den ersten Monaten zu beobachten waren. Diese Interpretation der Befunde wurde kontrovers diskutiert [20].

Dennoch legen die mit vergleichbaren Patientengruppen und unterschiedlichen Substanzen erzielten Ergebnisse nahe, dass eine inhalative Triple-Therapie gegenüber dualer Bronchodilatation in erster Linie bei symptomatischen $\mathrm{Pa}$ tienten mit einer Anamnese für häufige und/oder schwere Exazerbationen einen günstigen Effekt auf die Mortalität haben könnte.

Internist $2021 \cdot 62: 679-685$ https://doi.org/10.1007/s00108-021-01021-0

(c) Der/die Autor(en) 2021

\section{H. Olschewski • R. Buhl · G. C. Funk · A. Valipour · C. F. Vogelmeier}

\section{Chronisch-obstruktive Lungenerkrankung 2021 - die richtige Therapie für den richtigen Patienten}

\section{Zusammenfassung}

In den vergangenen Jahren wurden auf dem Gebiet der chronisch-obstruktiven Lungenerkrankung (COPD) große Therapiestudien publiziert, die 2020 um mehrere Post-hoc-Analysen ergänzt wurden. Die neuen Erkenntnisse flossen in das Update des Global-Initiative-for-Chronic-ObstructiveLung-Disease(GOLD)-Report 2021 ein. Im vorliegenden Beitrag werden die aktualisierten Grundlagen und Empfehlungen zur Therapie der COPD beschrieben. Dabei wird auf die Indikationen von inhalativen Kortikosteroiden (ICS), lang wirksamen Muskarinantagonisten (LAMA) und/oder lang wirksamen $\beta$-Mimetika (LABA) eingegangen. Die Therapie der COPD wird der des Asthma bronchiale gegenübergestellt. Diskutiert wird auch, wie sich eine gleichzeitig bestehende Asthmakomponente auf die
Behandlungsstrategie bei COPD auswirkt. Ein Schwerpunkt des Beitrags liegt auf der TripleTherapie mit LAMA, LABA und ICS. In diesem Zusammenhang werden die Studienlage und die Indikationen beschrieben. Die Bronchodilatation bleibt weiterhin die Grundlage der COPD-Therapie. Für Patienten mit gehäuften Exazerbationen bedeutet eine Triple-Therapie mit LAMA + LABA + ICS einen Mortalitätsvorteil. Weitere Analysen oder Studien sollen klären, ob dieser Effekt für spezifische Subgruppen stärker ausgeprägt ist.

\section{Schlüsselwörter}

Inhalative Kortikosteroide · Lang wirksame Muskarinantagonisten · Lang wirksame $\beta$-Mimetika - Asthma bronchiale . Exazerbation

\section{Chronic obstructive pulmonary disease: the right treatment for the right patient}

\section{Abstract}

In recent years, large treatment studies have been published in the field of chronic obstructive pulmonary disease (COPD) and were supplemented by several post-hoc analyses in 2020. The new evidence was incorporated into the 2021 update of the Global Initiative for Chronic Obstructive Lung Disease (GOLD) report. This article describes the updated fundamentals of and recommendations for the treatment of COPD. Indications for inhaled corticosteroids (ICS), long-acting muscarinic antagonists (LAMA), and/or long-acting beta-mimetics (LABA) will be addressed. The treatment of COPD is contrasted with that of bronchial asthma. The impact of a concomitant asthma component on the treatment strategy for
COPD is also discussed. In addition, the article focuses on triple therapy with LAMA, $\angle A B A$, and ICS, for which the evidence and indications are described. Bronchodilation remains the foundation of COPD therapy. For patients with clustered exacerbations, triple therapy with LAMA + LABA + ICS confers a mortality benefit. Further analysis or studies are needed to clarify whether this effect is more pronounced for specific subgroups.

\section{Keywords}

Corticosteroids, inhaled · Muscarinic antagonists, long-acting - Adrenergic betaagonists, long-acting · Asthma, bronchial . Disease exacerbation

\section{Details und mögliche Mechanismen}

In die IMPACT-Studie konnten COPDPatienten mit einer Asthmaanamnese eingeschlossen werden; Patienten mit deutlich eingeschränkter Lungenfunktion und sehr hohem Exazerbationsrisiko waren überrepräsentiert. Der abrupte Entzug von ICS bei Randomisierung in den LABA + LAMA-Studienarm kann gerade bei Patienten mit (subklinischer) asthmatischer Komponente trotz klinisch führender COPD dieStudienergebnisse maßgeblich beeinflussen $[19,21]$. Diese Annahme wird im Umkehrschluss durch Ergebnisse der IMPACT-Studie unterstützt, die keinen Nutzen einer ICS-Therapie bei Patienten zeigte, die vor Studieneinschluss keine ICS-The- 


\section{Arzneimitteltherapie}

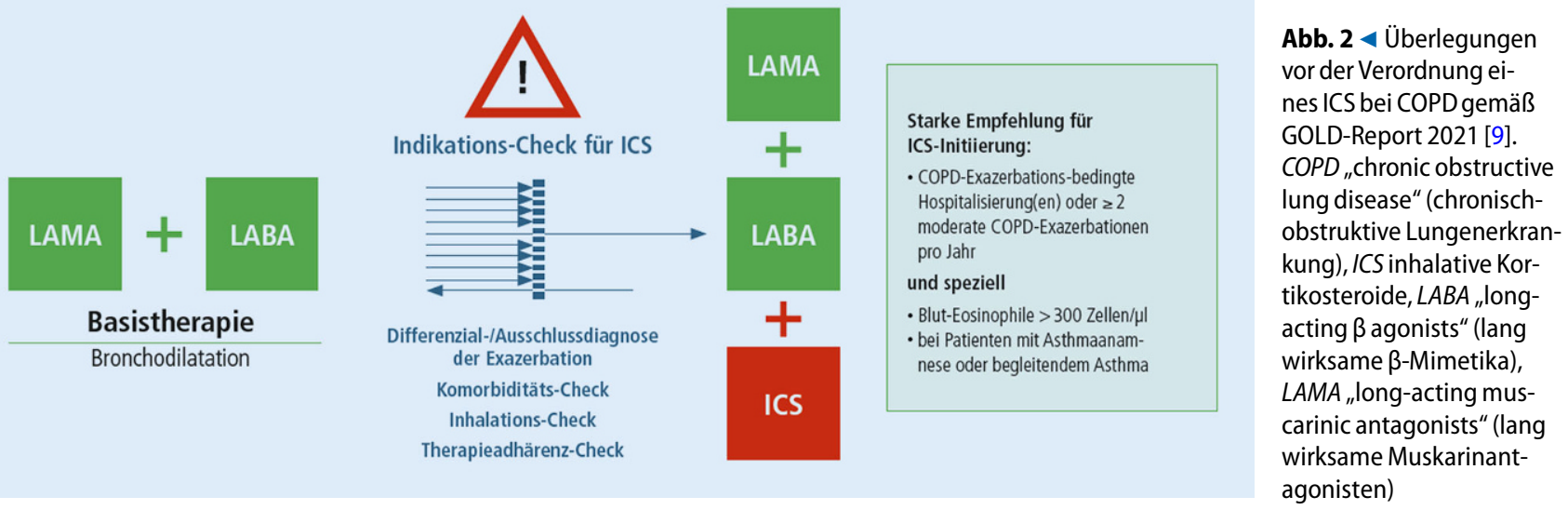

rapie hatten. In dieser Subgruppe war die Mortalität unter Triple-Therapie vergleichbar mit der Mortalität unter einer dualen Bronchodilatation (Hazard Ratio 1,25 ; $95 \%$-Konfidenzintervall [KI] $0,60-2,59)$, in deutlichem Unterschied zu Patienten mit einer ICS-Therapie vor Studieneinschluss (Hazard Ratio 0,63; $95 \%$-KI 0,44-0,89).

\section{》) Das abrupte Absetzen von ICS vor Studienbeginn kann die Studienergebnisse maßgeblich beeinflussen}

Die niedrige Mortalität unter TripleTherapie bei Patienten mit einer ICSVortherapie im Kontrast zum frühen und deutlichen Mortalitätssignal bei Randomisierung im LAMA + LABAStudienarm passt zu der Annahme, dass es der Entzug der ICS-Therapie ist, der die Mortalität entscheidend beeinflusst. Dazu passt auch die nahezu identische Mortalität in dieser Population in den Triple- und ICS + LABA-Studienarmen $[7,22,23]$.

\section{Rolle eines subklinischen Asthma bronchiale}

Es ist seit Langem bekannt, dass bei Patienten mit (subklinischer) asthmatischer Komponente trotz klinisch führender COPD, also bei Patienten mit einer anamnestischen Asthmaerkrankung und/oder asthmatypischem Symptomprofil (u.a. hohe Exazerbationsfrequenz, erhöhte Eosinophilenzahlen im peripheren Blut, bronchiale Hyperreagibilität; [24, 25]) und ICS-Therapie durch vorbehandelnde Ärzte, ein abrupter ICS-Entzug einen in doppelter Hinsicht negativen Einfluss auf die Mortalität hat. So erhöht die Beendigung einer ICS-Therapie bei Patienten mit Asthma die Mortalität innerhalb von nur 3 Monaten um mehr als den Faktor 4 („rate ratio“ 4,6; $95 \%$-KI 1,1-19,1; [19, 21, 26]). Umgekehrt führt eine Behandlung von Asthmapatienten nur mit einem LABA in gleicher Weise zu einer erhöhten Mortalität (Serevent Nationwide Surveillance Study: „rate ratio" 3,0; $95 \%$-KI 0,7-20,0; Salmeterol Multicenter Asthma Research Trial: „rate ratio“ 4,4; $95 \%$-KI 1,2-15,3; [27, 28]). Angesichts der Tatsache, dass bei $70 \%$ der in der IMPACT-Studie in den LAMA/LABA-Arm randomisierten $\mathrm{Pa}$ tienten eine ICS-Vortherapie plötzlich beendet wurde, erscheinen diese Punkte umso relevanter.

\section{Typ-2-Signatur oder eosinophile COPD?}

Asthma und COPD sind Erkrankungen mit unterschiedlichen Ätiologien, die allerdings gleichzeitig bei einem Patienten vorkommen können. Patienten mit COPD und gleichzeitigem Asthma haben eine deutlich höhere Exazerbationsfrequenz und deutlich schwerere Exazerbationen [29]. Etwa $20 \%$ der COPD-Patienten haben zusätzlich eine typische Asthmacharakteristik bzw. eine Typ-2Signatur [30,31]. Dafür sprechen erhöhte Eosinophilenzahlen im Blut und im Sputum und erhöhte Werte der Fraktion des exhalierten Stickstoffmonoxids (FeNO) sowie eine allergische Diathese des $\mathrm{Pa}$ tienten oder seiner Familie. Auch eine subklinische Typ-2-Signatur kann sich in der Exazerbation demaskieren. So wiesen $28 \%$ der Patienten mit COPD-Exazerbation im Sputum und im Blut Charakteristika von Patienten mit Asthmaexazerbation auf [32]. Ob auch die bronchiale Hyperreagibilität für die Differenzialindikation eine Bedeutung hat, kann nicht abschließend beurteilt werden.

\section{Rolle des Zigarettenrauchens}

Das Rauchen spielt sowohl für das Asthma als auch für die COPD eine wichtige Rolle: Der Schweregrad eines Asthmas kann durch Rauchen gesteigert werden, bei gleichzeitig vermindertem Ansprechen auf eine ICS-Therapie. Es kommt bei Rauchexposition zu einer stärkeren Obstruktion und mehr Exazerbationen [33]. Die COPD ist neben den Gefäßerkrankungen und Lungenkrebs bekanntermaßen die häufigste Folgekrankheit des Rauchens. Durch das Beenden des Rauchens verlangsamt sich der jährliche Abfall der Lungenfunktion. Bei Asthma führt das Zigarettenrauchen früher $\mathrm{zu}$ einer fixierten Obstruktion als bei Patienten ohne Asthma. Daher weisen die Asthmatiker unter den COPD-Patienten besonders wenig Packungsjahre auf. Umgekehrt ist bei COPD-Patienten kein Ansprechen auf ICS nachweisbar, wenn $<2,4 \%$ Eosinophile und $>46$ Packungsjahre vorliegen [16]. Das bedeutet, dass auch eine niedrige Zahl von Packungsjahren beim COPD-Patienten einen wichtigen Hinweis auf eine Asthmakomponente darstellen kann. 
In Summe machen diese Argumente deutlich, dass die Mortalitätssenkung im Zusammenhang mit einer Triple-Therapie bei COPD vermutlich auf Patienten beschränkt ist, die bestimmte Voraussetzungen mitbringen. Dazu zählen Patienten mit häufigen und/oder schweren Exazerbationen unter schon bestehender Therapie in Verbindung mit erhöhten Eosinophilenzahlen im peripheren Blut. Empfehlungen der GOLD und European Respiratory Society (ERS) ebenso wie aktuelle Metaanalysen stützen diese Annahme [34-37]. Die Bedeutung einer möglichen Asthmakomponente für diese Konstellation bleibt zu klären.

\section{Fazit für die Praxis}

- Die Bronchodilatation bleibt die Grundlage der Therapie bei chronisch-obstruktiver Lungenerkrankung (COPD).

- Die wissenschaftliche Evidenz unterstützt die Empfehlung, die Gabe von Kombinationstherapien mit inhalativen Kortikosteroiden den Patienten mit COPD und vermehrten Exazerbationen vorzubehalten. Abgesehen von häufigen und/oder schweren Exazerbationen sind die Patienten klinisch charakterisiert durch erhöhte Eosinophilenzahlen im Blut.

- Eine Exazerbation ist definiert als eine akute Verschlechterung der respiratorischen Symptome, die zu einer Steigerung der Therapie führt. Doch nicht jede Verschlechterung kommt durch eine Exazerbation zustande.

- Eine niedrige Zahl von Packungsjahren beim COPD-Patienten kann ein wichtiger Hinweis auf eine Asthmakomponente sein.

\section{Korrespondenzadresse}

Univ.-Prof. Dr. Horst Olschewski

Klinische Abteilung für Pulmonologie,

Universitätsklinik für Innere Medizin,

Medizinische Universität Graz

Auenbruggerplatz 15, 8036 Graz, Österreich

horst.olschewski@medunigraz.at

Funding. Open access funding provided by Medical University of Graz.

\section{Einhaltung ethischer Richtlinien}

Interessenkonflikt. Die Autoren weisen auf folgende Beziehungen hin. H. Olschewski erhielt Honorare für Vortrags- und Beratertätigkeiten von Actelion, AstraZeneca, Bayer, Boehringer Ingelheim, Chiesi, GlaxoSmithKline, Inventiva, Janssen, MedUpdate, Menarini, MSD, Novartis und Pfizer und Forschungsunterstützung für die Medizinische Universität Graz durch Boehringer Ingelheim, Roche und Inventiva. Er ist stv. Direktor des Ludwig Boltzmann Instituts für Lungengefäßforschung. R. Buhl erhielt Honorare für Vortragsund Beratertätigkeiten von AstraZeneca, Berlin-Chemie, Boehringer Ingelheim, Chiesi, GlaxoSmithKline, Novartis und Sanofi. Forschungsunterstützung an die Universitätsmedizin Mainz durch AstraZeneca, Boehringer Ingelheim, Bosch, GlaxoSmithKline, Novartis, Roche und Sanofi. G.C. Funk erhielt Honorare für Vortrags- und/oder Beratertätigkeiten und/oder Forschungsunterstützung von AstraZeneca, Boehringer Ingelheim, Chiesi, Menarini und Novartis. A. Valipour erhielt Honorare für Vortrags- und/oder Beratertätigkeiten von AstraZeneca, Boehringer Ingelheim, Chiesi, Menarini, Merck, Novartis, und Takeda. C.F. Vogelmeier erhielt Honorare für Vortrags- und Beratertätigkeiten von AstraZeneca, Boehringer Ingelheim, CSL Behring Chiesi, GlaxoSmithKline, Grifols, Menarini, Novartis, Nuvaira und MedUpdate. Die Idee zu der Publikation entstand im Rahmen eines Advisory Board Meetings von Boehringer Ingelheim.

Für diesen Beitrag wurden von den Autoren keine Studien an Menschen oder Tieren durchgeführt. Für die aufgeführten Studien gelten die jeweils dort angegebenen ethischen Richtlinien.

Open Access. Dieser Artikel wird unter der Creative Commons Namensnennung 4.0 International Lizenz veröffentlicht, welche die Nutzung, Vervielfältigung, Bearbeitung, Verbreitung und Wiedergabe in jeglichem Medium und Format erlaubt, sofern Sie den/die ursprünglichen Autor(en) und die Quelle ordnungsgemäß nennen, einen Link zur Creative Commons Lizenz beifügen und angeben, ob Änderungen vorgenommen wurden.

Die in diesem Artikel enthaltenen Bilder und sonstiges Drittmaterial unterliegen ebenfalls der genannten Creative Commons Lizenz, sofern sich aus der Abbildungslegende nichts anderes ergibt. Sofern das betreffende Material nicht unter der genannten Creative Commons Lizenz steht und die betreffende Handlung nicht nach gesetzlichen Vorschriften erlaubt ist, ist für die oben aufgeführten Weiterverwendungen des $\mathrm{Ma}$ terials die Einwilligung des jeweiligen Rechteinhabers einzuholen.

Weitere Details zur Lizenz entnehmen Sie bitte der Lizenzinformation auf http://creativecommons.org/ licenses/by/4.0/deed.de.

\section{Literatur}

1. Singh D et al (2016) Single inhaler triple therapy versus inhaled corticosteroid plus long-acting beta2-agonist therapy for chronic obstructive pulmonary disease (TRILOGY): a double-blind, parallel group, randomised controlled trial. Lancet 388(10048):963-973

2. Vestbo J et al (2017) Single inhaler extrafine triple therapy versus long-acting muscarinic antagonist therapy for chronic obstructive pulmonary disease (TRINITY): a double-blind, parallel group, randomised controlled trial. Lancet 389(10082):1919-1929

3. Papi $A$ et al (2018) Extrafine inhaled triple therapy versus dual bronchodilator therapy in chronic obstructive pulmonary disease (TRIBUTE): a double-blind, parallel group, randomised controlled trial. Lancet 391(10125):1076-1084

4. Lipson DA et al (2018) Once-daily single-inhaler triple versus dual therapy in patients with COPD. N Engl J Med 378(18):1671-1680

5. Vestbo J et al (2016) Fluticasone furoate and vilanterol and survival in chronic obstructive pulmonary disease with heightened cardiovascular risk (SUMMIT): a double-blind randomised controlled trial. Lancet 387(10030):1817-1826

6. Rabe KF et al (2020) Triple inhaled therapy at two glucocorticoid doses in moderate-to-very-severe COPD. NEngl JMed 383(1):35-48

7. Lipson DA et al (2020) Reduction in all-cause mortality with fluticasone furoate/umeclidinium/ vilanterol in patients with chronic obstructive pulmonary disease. Am J Respir Crit Care Med 201(12):1508-1516

8. Martinez FJ, Rabe KF, Ferguson GT, Wedzicha JA, Singh D, Wang C, Rossman K, St. Rose E, Trivedi R, Ballal S, Darken P, Aurivillius M, ReisnerC, DorinskyP (2021) Reduced all-cause mortality in the ETHOS trial of budesonide/glycopyrrolate/formoterol for COPD: a randomized double-blind, multi-center parallel-group study. Am J Respir Crit Care Med 203(5):553-564. https://doi.org/10.1164/rccm. 202006-26180C

9. GOLD (2021) Global strategy for prevention, diagnosis and management of COPD. https:// goldcopd.org/gold-reports/.Zugegriffen:21. Feb. 2021

10. Couturaud $\mathrm{F}$ et al (2021) Prevalence of pulmonary embolism among patients with COPD hospitalized with acutely worsening respiratory symptoms. JAMA 325(1):59-68

11. Oba Y et al (2018) Dual combination therapy versus long-acting bronchodilators alone for chronic obstructive pulmonary disease (COPD): a systematic review and network meta-analysis. Cochrane Database Syst Rev 12:CD12620

12. Maltais F et al (2019) Efficacy of umeclidinium/ vilanterol versus umeclidinium and salmeterol monotherapies in symptomatic patients with COPD not receiving inhaled corticosteroids: the EMAX randomised trial. Respir Res 20(1):238

13. Vogelmeier $C F$, Boucot $I H$, Kerwin EM, Bjermer L, Jones PW, Naya IP, Tombs L, Compton C, Lipson DA, Maltais F (2020) Improvements in COPD symptoms with umeclidinium/vilanterolanalyzed by baseline CAT score: a post hoc analysis of the EMAX trial. American Thoracic Society, SA3325

14. Kew KM, Dahri K (2016) Long-acting muscarinic antagonists (LAMA) added to combination longacting beta2-agonists and inhaled corticosteroids (LABA/ICS) versus LABA/ICS for adults with asthma. Cochrane Database Syst Rev 1:CD11721

15. Celli BR et al (2021) Pharmacotherapy and lung function decline in patients with chronic obstructive pulmonary disease: a systematic review. Am J Respir Crit Care Med 203(6):689-698. https://doi.org/10.1164/rccm.202005-18540C

16. Hinds DR et al (2016) Identification of responders to inhaled corticosteroids in a chronic obstructive pulmonary disease population using cluster analysis. BMJ Open 6(6):e10099 
17. GINA (2020) Global strategy for asthma management and prevention (updated 2020). https:// www.ginasthma.org.Zugegriffen:21. Feb. 2021

18. Vogelmeier C et al (2018) Guideline for the diagnosisand treatment ofCOPDpatients-issued by the German respiratory society and the German Atemwegsliga in cooperation with the Austrian society of pneumology. Pneumologie 72(4):253-308

19. Suissa S, Ariel A (2018) Triple therapy trials in COPD: a precision medicine opportunity. Eur Respir J 52(6):1801848. https://doi.org/10.1183/ 13993003.01848-2018

20. Papi Aetal (2019) Triple therapy for all patients with severe symptomatic COPD at risk of exacerbations. Eur Respir J 53(4):1900147. https://doi.org/10. 1183/13993003.00147-2019

21. Suissa S, Drazen JM (2018) Making sense of triple inhaled therapy for COPD. N Engl J Med 378(18):1723-1724

22. Suissa S (2020) Mortality in IMPACT: confounded by asthma? Am J Respir Crit Care Med 202(5):772-773

23. Lipson DA, Dransfield MT, Han MK (2020) Reply to Suissa: mortality in IMPACT: confounded by asthma? Am J Respir Crit Care Med 202(5):773-774

24. Hospers JJ et al (2000) Histamine airway hyperresponsiveness and mortality from chronic obstructive pulmonary disease: a cohort study. Lancet 356(9238):1313-1317

25. Teferra AA, Vonk JM, Boezen HM (2020) Longitudinal changes in airway hyperresponsiveness and COPD mortality. Eur Respir J 55(2):1901378. https://doi.org/10.1183/13993003.01378-2019

26. Suissa S et al (2000) Low-dose inhaled corticosteroids and the prevention of death from asthma. NEngl J Med 343(5):332-336

27. Castle $W$ et al (1993) Serevent nationwide surveillance study: comparison of salmeterol with salbutamol in asthmatic patients who require regular bronchodilator treatment. BMJ 306(6884):1034-1037

28. Nelson HS et al (2006) The salmeterol multicenter asthma research trial: a comparison of usual pharmacotherapy for asthma or usual pharmacotherapy plus salmeterol. Chest 129(1):15-26

29. Hardin M et al (2011) The clinical features of the overlap between COPD and asthma. Respir Res $12: 127$

30. Miravitlles M et al (2015) What pulmonologists think about the asthma-COPD overlap syndrome. Int JChron Obstruct Pulmon Dis 10:1321-1330

31. Christenson SA et al (2015) Asthma-COPD overlap. Clinical relevance of genomic signatures of type 2 inflammation in chronic obstructive pulmonary disease. Am J Respir Crit Care Med 191(7):758-766

32. Bafadhel Metal (2011) Procalcitonin and C-reactive protein in hospitalized adult patients with community-acquired pneumonia or exacerbation of asthma or COPD. Chest 139(6):1410-1418

33. GINA (2019) Difficult-to-treat and severe asthma in adolescents and adults. ginasthma.org/ severeasthma/.Zugegriffen: 2021

34. Mammen MJ etal (2020) Triple therapy versus dual or monotherapy with long-acting bronchodilators for chronic obstructive pulmonary disease. A systematic review and meta-analysis. Ann Am Thorac Soc 17(10):1308-1318

35. Chalmers JD et al (2020) Withdrawal of inhaled corticosteroids in COPD: a European respiratory society guideline. Eur Respir J 55(6):2000351. https://doi.org/10.1183/13993003.00351-2020

36. Stolz D, Miravitlles M (2020) The right treatment for the right patient with COPD: lessons from the
IMPACT trial. Eur Respir J 55(5):2000881. https:// doi.org/10.1183/13993003.00881-2020

37. Suissa S, Dell'Aniello S, Ernst P (2020) Comparative effects of LAMA-LABA-ICS vs LAMA-LABA forCOPD: cohort study in real-world clinical practice. Chest 157(4):846-855
Operationen nach SARSCoV-2-Infektion

Eine neue weltweite Studie des Forschungsnetzwerks COVIDSurg, an dem auch die Universitätsmedizin Halle (Saale) beteiligt ist, zeigt: Operationen sollten nach einer Infektion mit dem Corona-Virus SARS-CoV-2 erst mindestens sieben Wochen nach dem Nachweis stattfinden.

Bereits im Mai 2020 belegten Daten von COVIDSurg, dass die Sterblichkeit von Patientinnen und Patienten bei Operationen nach Coronavirus-Infektionen erhöht ist. In einer aktuellen Veröffentlichung der Fachzeitschrift Anaesthesia nennt das Forschungsnetzwerk nun einen konkreten Zeitraum: Betroffene haben während der ersten sechs Wochen nach einem SARSCoV-2-Nachweis verglichen mit einem später stattfindenden Eingriff ein über zweieinhalbfach erhöhtes Risiko, nach der Operation zu versterben.

Operationen sollten daher entsprechend aufgeschoben werden. Dies gilt auch, wenn nach den sieben Wochen die COVID-19-Symptomatik noch andauert. Bei dringlichen Eingriffen jedoch, wie etwa Tumoroperationen, muss das Risiko eines Fortschreitens der Erkrankung streng gegen das erhöhte Operationsrisiko abgewogen und in bestimmten Fällen auch früher operiert werden.

Das internationale Forschungsnetzwerk COVIDSurg unter der Leitung der Universität Birmingham umfasst über 25.000 Chirurginnen und Chirurgen sowie Wissenschaftlerinnen und Wissenschaftler. Das Projekt ist eine der größten klinischen Studien, die jemals durchgeführt wurden.

Quelle: Universitätsmedizin Halle (Saale), www.medizin.uni-halle.de 
Hier steht eine Anzeige.

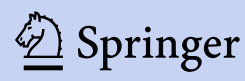

